PROCEEDINGS OF THE

AMER!CAN MATHEMATICAL SOCIETY

Volume 33. Number 2, June 1972

\title{
ON PERFECT SUBFIELDS OVER WHICH \\ A FIELD IS SEPARABLY GENERATED
}

DAVID JACOBSON

\begin{abstract}
In this paper, we determine the perfect subfields over which a field is separably generated.
\end{abstract}

Let a field $F$ of characteristic $p \neq 0$ be an extension of a field $E$. A separating transcendence basis for the extension $F / E$ is a transcendence basis $B$ of $F / E$ such that $F$ is a separable algebraic extension of $E(B)$. If $F / E$ has a separating transcendence basis, we say that $F$ is separably generated over $E$.

A field $K$ of characteristic $p \neq 0$ is perfect if $K^{p}=K$, that is, if each element of $K$ has a unique $p$ th root in $K$. It is clear that any field $F$ contains a maximal perfect subfield which we denote by $F^{*}$ and that $F^{*}=\bigcap_{n=1}^{\infty} F^{p^{n}}$.

We prove in Theorem 3 that if $F$ is separably generated over a subfield $E$, then $F^{*}$ is an algebraic extension of $E^{*}$.

As a consequence (Corollary 10 ), we show that if a field $F$ is separably generated over at least one perfect subfield, then $F$ is separably generated over a perfect subfield $K$ if and only if $F^{*}$ is algebraic over $K$.

LeMma 1. If $F$ is a pure transcendental extension of $E$, then $F^{*}=E^{*}$.

Proof. By hypothesis, $F=E(B)$ where $B$ is an algebraically independent set of elements over $E$. If $y$ is a nonzero element of $F^{*}$, then there exist elements $w_{n}$ in $F$ such that $y=w_{n}^{p^{n}}$ for $n=1,2, \cdots$. Since the polynomial ring $E[B]$ is a unique factorization domain, we may write $y=f / g$ with $f$ and $g$ relatively prime in $E[B]$. Similarly, for each $n, w_{n}=f_{n} / g_{n}$ with $f_{n}$ and $g_{n}$ relatively prime in $E[B]$. Thus $f g_{n}^{p^{n}}=g f_{n}^{\nu^{n}}$ implies that $f$ and $f_{n}^{p^{n}}$ are associates for each $n$, that is, $f=a_{n} f_{n}^{x^{n}}$ where $a_{n}$ is in $E$. As a consequence of the unique factorization in $E[B]$, it follows that $f \in E$ and $f_{n} \in E$, for all $n$. Similarly $g \in E$ and $g_{n} \subseteq E$, for all $n$. Thus $y \in E^{*}=$ $\bigcap_{n=1}^{\infty} E^{p^{n}}$, proving that $F^{*}=E^{*}$.

The following lemma appears as an exercise in [1, Example 16, p. 136]. For the sake of completeness, we provide a proof.

Presented to the Society. January 17, 1972; received by the editors September 29, 1971.

AMS 1970 subject classifications. Primary 12E05, 12F05, $12 \mathrm{~F} 20$.

Key words and phrases. Separable algebraic extension, separating transcendence basis, separable generation, perfect field, maximal perfect subfield.

(c) American Mathematical Society 1972 
LEMMA 2. Let $f(x)$ be a monic irreducible polynomial in $E[x]$, where $E$ is a field of characteristic $p \neq 0$. Then in $E[x]$, the polynomial $f\left(x^{p}\right)$ is irreducible or is the pth power of an irreducible polynomial according to whether or not there exists a coefficient of $f(x)$ which does not belong to $E^{p}$.

Proof. Let $F$ be an extension field of $E$ which contains a root $w$ of $g(x)=f\left(x^{p}\right)$. If $u=w^{p}$, then $f(u)=g(w)=0$. Thus the degree of $E(u)$ over $E$ is equal to $m$, the degree of $f(x)$, since $f(x)$ is irreducible in $E[x]$. Suppose that $g(x)$ is reducible in $E[x]$. Then $E(w)=E(u)$, for $w$ not in $E(u)$ implies that the degree of $E(w)$ over $E(u)$ is $p[3$, Theorem 7, p. 66] and so the degree of $E(w)$ over $E$ is $p m$, contradicting the reducibility of $g(x)$. Thus if $h(x)=x^{m}+b_{1} x^{m-1}+\cdots+b_{m}$ is the minimum polynomial of $w$ over $E$, then $h(w)=0$ and $(h(w))^{p}=0$, that is, $u^{m}+b_{1}^{p} u^{i n-1}+\cdots+b_{m}^{p}=0$. Therefore, $f(x)=x^{m}+b_{1}^{x} x^{m-1}+\cdots+b_{m}^{p}$ and $f\left(x^{p}\right)=(h(x))^{p}$, completing the proof.

THEOREM 3. If a field $F$ is separably generated over a subfield $E$, then $F^{*}$ is an algebraic extension of $E^{*}$.

Proof. If $B$ is a separating transcendence basis of $F$ over $E$, then $F$ is a separable algebraic extension of $F_{1}=E(B)$. Let $u$ be any element of $F^{*}$ and let $f(x)=x^{m}+a_{1} x^{m-1}+\cdots+a_{m}$ be the minimum polynomial of $u$ over $F_{1}$. There exists an element $w$ in $F^{*}$ such that $w^{p}=u$ and since $F$ is a separable algebraic extension of $F_{1}(u)$, it foilows that $w$ is in $F_{1}(u)$. If $g(x)=f\left(x^{p}\right)$, then $g(w)=0$ and hence $g(x)$ is reducible in $F_{1}[x]$. By Lemma 2, $g(x)=(h(x))^{p}$ where $h(x)=x^{m}+b_{1} x^{m-1}+\cdots+b_{m}$ is irreducible in $F_{1}[x]$. Thus $h(w)=0$ and $b_{i}^{p}=a_{i}$, for $i=1, \cdots, m$. By repeating the argument for $w$, we obtain an element $w_{1}$ in $F^{*}$ with $w_{1}^{p}=w^{\prime}$ and an irreducible polynomial $h_{1}(x)=x^{m}+c_{1} x^{m-1}+\cdots+c_{m}$ in $F_{1}[x]$ such that $h_{1}\left(w_{1}\right)=0$ and $c_{i}^{p}=b_{i}$, for $i=1, \cdots, m$. Continuing inductively, we have for each $i$, a sequence of elements $d(i, n)$ in $F_{1}$ such that $d_{(i, n)}^{p^{n}}=a_{i}$, that is, $a_{i} \in F_{1}^{*}=$ $\bigcup_{n=1}^{\infty} F_{1}^{p^{n}}$, for $i=1, \cdots, m$. However Lemma 1 shows that $F_{1}^{*}=E^{*}$, and so $f(x) \in E^{*}[x]$. Hence $u$ is algebraic over $E^{*}$ which completes the proof.

EXAMPLE. The following example shows that the equality $F^{*}=E^{*}$ does not always hold given only the hypothesis of Theorem 3 . If $E$ is the finite field with $p$ elements and $F$ is a simple transcendental extension of the field with $p^{2}$ elements, then by [2, Theorem 5], $F$ is separably generated over $E$, although $F^{*} \neq E^{*}$.

We shall also require the following result.

THEOREM 4. Let $K$ be an extension field of $E$ and $F$ an extension of $K$. The following statements are equivalent.

(i) $F$ is separably generated over $E$ and $K$ is an algebraic extension of $E$.

(ii) $F$ is separably generated over $K$ and $K$ is a separable algebraic extension of $E$. 
Proof. Assume that (i) holds and let $B$ be a separating transcendence basis of $F$ over $E$. We show that $B$ is also a separating transcendence basis of $F$ over $K$. As $F$ is a separable algebraic extension of $E(B)$ and thus a separable algebraic extension of $K(B)$, it remains to prove that $B$ is a transcendence basis of $F$ over $K$. However, $B$ contains a transcendence basis $B^{\prime}$ of $F$ over $K$ and since $K$ is assumed to be algebraic over $E$, we see that $K\left(B^{\prime}\right)$ is algebraic over $E\left(B^{\prime}\right)$ and so $K(B)$ is algebraic over $E\left(B^{\prime}\right)$. This implies that $E(B)$ is an algebraic extension of $E\left(B^{\prime}\right)$ and hence $B=B^{\prime}$. Moreover, $K$ is a separable algebraic extension of $E$ since $K(B)$ is a separable algebraic extension of $E(B)$.

Conversely, if (ii) holds, then $K$ is clearly separably generated over $E$ and transitivity of separable generation implies that $F$ is separably generated over $E$.

For a subfield $E$ of a field $F$, let $E_{0}$ denote the subfield of $F$ of elements separable algebraic over $E$ and $\vec{E}$ the algebraic closure of $E$ in $F$.

Corollary 5. $F$ is separably generated over $E$ if and only if $F$ is separably generated over $E_{0}$. If either (and hence both) of these conditions holds, then $E_{0}=\bar{E}$.

Proof. If $F$ is separably generated over $E$, then Theorem 4 shows that $E_{0}=\bar{E}$ and $F$ is separably generated over $E_{0}$. Conversely if $F$ is separably generated over $E_{0}$, then $F$ is separably generated over $E$.

We remark that the example shows that [2, Theorem 19] is incorrect as stated. As Professor Mac Lane has noted in a personal communication, [2, Theorem 19] becomes valid with the additional hypothesis that $E$ is algebraically closed in $F$. We now recover this result from the next corollary.

Corollary 6. If a field $F$ has a separating transcendence basis over a subfield $E$, then $E_{0}$ contains $F^{*}$.

Proof. By Theorem $3, F^{*}$ is a separable algebraic extension of $E^{*}$ and thus $E_{0}$ contains $F^{*}$.

COROLlARY 7 (MAC LANE). If a field $F$ has a separating transcendence basis over a subfield $E$ and $E$ is algebraically closed in $F$, then $E$ contains the maximal perfect subfield of $F$.

COROLlaRY 8. If $F / K$ and $K / E$ are field extensions, then the following are equivalent.

(1) $F$ is separably generated over $E$ and $K$ is perfect.

(2) $E$ is perfect, $K$ is an algebraic extension of $E$ and $F$ is separably generated over $K$. 
Proof. If (1) holds, then Theorem 3 shows that $F^{*}$ is algebraic over $E^{*}$. Since $K$ is perfect, $F^{*}$ is an extension of $K$. Thus by Theorem $4, E$ is perfect since $K$ is a separable algebraic extension of $E$ and $F$ is separably generated over $K$.

Conversely if (2) holds, then $K$ is a separable algebraic extension of $E$ and $K$ is perfect. Applying Theorem 4 , we find that $F$ is separably generated over $E$, to complete the proof.

For example, if $E$ is a perfect field and $K=E\left(x, x^{p^{-1}}, x^{p^{-2}}, \cdots\right)$ where $x$ is transcendental over $E$, then no extension field of $K$ is separably generated over $E$.

COROLlaRY 9. Let $F$ be an extension field of $E$ and suppose that $F$ is separably generated over $E$. If $E$ is separably generated over $E^{*}$, then $F$ is separably generated over $F^{*}$. Conversely, if $F$ is separably generated over $F^{*}$ and $E$ has finite transcendence degree over $E^{*}$, then $E$ is separably generated over $E^{*}$.

Proof. If $E$ is separably generated over $E^{*}$, then by transitivity, $F$ is separably generated over $E^{*}$. Hence $F$ is separably generated over $F^{*}$ by Corollary 8 .

For the converse, note $F^{*}$ is algebraic over $E^{*}$ by Theorem 3 . If $F$ is separably generated over $F^{*}$, then Corollary 8 implies that $F$ is separably generated over $E^{*}$. Further, if $\operatorname{tr}$. d. $E_{l}^{\prime} E^{*}$ is finite, then $E$ is separably generated over $E^{*}$ by the corollary in $[2$, p. 386].

COROLlaRY 10. Let $F$ be a field and suppose that the set $\Omega$ of perfect subfields over which $F$ is separably generated is not empty. Then $F^{*}$ belongs to $\Omega$. Moreover, a perfect subfield $E$ belongs to $\Omega$ if and only if $F^{*}$ is an algebraic extension of $E$.

PRoof. It is evident from Corollary 9 that $F^{*}$ belongs to $\Omega$ and hence Corollary 8 establishes the assertion.

For a given field $F$, the set $\Omega$ may be empty. Indeed Mac Lane $[2, \S 10]$ has shown the existence of a field $M$ which does not have a separating transcendence basis over its maximal perfect subfield $M^{*}$, although tr. d. $M / M^{*}=2$. However, if a field $F$ has transcendence degree 1 or is finitely generated over $F^{*}$, then $F$ is always separably generated over $F^{*}$ [2, Theorems 1 and 4$]$.

We ask whether there exists an imperfect field which is separably generated only over itself. Such a field satisfies this property if and only if it is separable algebraic only over itself, since any pure transcendental extension is a separable algebraic extension of a proper subextension.

We close by stating the following consequence of Corollary 8 . 
COROLlaRY 11. Let $F$ be a field which is separably generated over its prime subfield $P$. If $K$ is any perfect subfield of $F$, then $K$ is an algebraic extension of $P$ and $F$ is separably generated over $K$.

\section{REFERENCES}

1. N. Bourbaki, Eléments de mathématique. I: Les structures fondamentales de l'analyse. Fasc. XI. Livre II: Algèbre. Chaps. 4, 5, Actualités Sci. Indust., no. 1102, Hermann, Paris, 1959. MR 30 \#451.

2. S. Mac Lane, Separating transcendence bases, Duke Math. J. 5 (1939), 372-393.

3. O. Zariski and P. Samuel, Commutative algebra. Vol. I, University Series in Higher Math., Van Nostrand, Princeton, N.J., 1957. MR 19, 833.

Department of Mathematics, Rutgers University, New Brunswick, New JERSEY 08903 